\section{EMBRYRIDDLE \\ Aeronautical University}

SCHOLARLY COMMONS
International Journal of Aviation, Aeronautics, and Aerospace

\title{
Adaptive filtration of the UAV movement parameters based on the AOA-measurement sensor networks
}

Igor Tovkach

National Technical University of Ukraine "Igor Sikorsky Kyiv Polytechnic Institute",

tovkach.igor@gmail.com

Serhii Zhuk

National Technical University of Ukraine "Igor Sikorsky Kyiv Polytechnic Institute", serya58@ukr.net

Follow this and additional works at: https://commons.erau.edu/ijaaa

Part of the Navigation, Guidance, Control and Dynamics Commons

\section{Scholarly Commons Citation}

Tovkach, I., \& Zhuk, S. (2020). Adaptive filtration of the UAV movement parameters based on the AOAmeasurement sensor networks. International Journal of Aviation, Aeronautics, and Aerospace, 7(3). https://doi.org/10.15394/ijaaa.2020.1497

This Article is brought to you for free and open access by the Journals at Scholarly Commons. It has been accepted for inclusion in International Journal of Aviation, Aeronautics, and Aerospace by an authorized administrator of Scholarly Commons. For more information, please contact commons@erau.edu. 
In recent years, small unmanned aerial vehicles (UAVs) (both mini and micro) have become a popular surveillance tool in the field of defense and security and constantly evolving technological progress, provides a brilliant future for this technology. The military also increased interest in small UAVs, which can be used to solve tactical reconnaissance, electronic warfare, laserguided various weapons platforms, or to deliver small bombs. In many countries of the world, continuous research and development work is being carried out in this direction. According to ICInsights, taking into account all potential areas of use, the global sales of devices in 2025 may exceed $\$ 10$ billion (2017).

On the other hand, the use of UAVs has led to new potential threats to national and public security. Such UAVs can carry explosives, biological, or chemical weapons to carry out terrorist acts. Devices can also be used to transport smuggling, drugs, jamming GPS signals or Wi-Fi, which will lead to interruption in communication and data transfer (Wallace \& Loffi, 2015).

The greatest vulnerability of UAVs is due to the presence of electromagnetic radiation. The standard radio frequency UAV bands are ISM $2.4 \mathrm{GHz}$ and ISM $5.8 \mathrm{GHz}$, at which operate most commercial Wi-Fi, Bluetooth and IoT systems (i.e. ZigBee, Z-Wave, LoRa). The signals in these bands are freely regulated using free access rules.

Recent advances in wireless sensor networks are opening up new possibilities in solving the problem of determining of radio sources location. Wireless sensor network is a set of miniature and inexpensive devices equipped with various types of sensors, a small microcontroller and a receiver, which are connected via a WLAN network and uses radio channels for data transmission (Chu \& Han,2019). This task has a wide range of applications, such as: rescue operations, autonomous surveillance and monitoring of industrial processes and the environment (monitoring of the animal world), monitoring and control of moving objects, etc.

One of the important features of wireless sensor networks is the ability to track moving objects (targets), including UAVs (Liu, Li \& Yang, 2018; Mohd $\&$ Rajesh, 2018). In recent years, a number of methods have been developed to determine an unknown RS location using WSN (Amiri et al. 2016; Chen \& Wu, 2018; Hou et al. 2018; Peng \& Sichitiu, 2006; Tomic et al. 2018; Tovkach \& Zhuk, 2017a; Tovkach et al. 2018; Tovkach et al. 2019; Zhang et al. 2012; Zhang et al. 2018; Zhuk et al. 2018, 2019): RSS (Received Signal Strength), ToA (Time of Arrival), TDoA (Time Difference of Arrival), AoA (Angle of Arrival).

The AOA method is among the oldest positioning methods. It is based on measuring the angular coordinates of the radio source using several reception points with known coordinates that are located at different points in space, as well as using trigonometric relations to determine the location of the radio source in space. The main difference of the AOA method is that it does not require synchronization with a radio source and between reception points (Yao et al. 2014; Zhang et al. 2013). The advantage of this method is the simplicity 
of the technical component of its application. Therefore, he found the widest application in practice.

When using the AOA method to determine the radio source spatial coordinates it is necessary to find the three angular coordinates of the radio source at different receiving points. It can be two azimuths and elevation, or one azimuth and two elevations. In this case, the radio source location will be determined as the intersection point of the three planes defined by these angles. Currently, smart and adaptive antenna arrays are widely used to determine the direction of arrival of a signal (Tang et al., 2007; Xu et al., 2008).

A feature of modern UAVs is the ability to perform sudden maneuvers, and keep the same position in the point in space. Changing of the type of UAV movement occurs in random, unknown to the observer, moments of time, and this allows to represent a trajectory in the form of stochastic process, the probability characteristics of which change by leap at random moments in time. A convenient mathematical model of such processes is stochastic discrete dynamic systems with random structure that are adequate to the tasks being solved when implementing algorithms on digital computers.

To improve the accuracy of UAV coordinates estimation in areas with different types of movement, it is necessary to use different measurement processing algorithms. However, the type of movement is usually unknown. Therefore, simultaneously with the task of estimating UAV coordinates, it is also necessary to solve the task of recognizing the type of its movement. At hanging intervals, as well as for UAV movement without maneuver, it is possible to increase significantly the accuracy of estimating its coordinates. Moreover, in practice it is also often of interest to determine the UAV movement types.

The article is devoted to the development of an adaptive estimation algorithm of UAV movement parameters based on AOA-measurements sensor networks. In Section 2, a mathematical formulation of the problem is formulated, in Section 3, the optimal algorithm of adaptive estimation of the UAV movement parameters are synthesized, in Section 4, Linearization of UAV coordinate measurement equations in a Cartesian coordinate system, in Section 5, the quasi-optimal algorithm of adaptive estimation of the UAV movement parameters are synthesized and in section 6 , the effectiveness of the developed algorithm is analyzed.

\section{Formulation of the Problem}

Wireless sensor network consists of $B$ pairs of sensors $S_{b i}, i=\overline{0,1}$. Without loss of consideration generality, we will assume that they are located on the horizontal plane $X Y$ with coordinates $\left(x_{b i}^{S}, y_{b i}^{S}\right), i=\overline{0,1}, b=\overline{1, B}$. Figure 1 


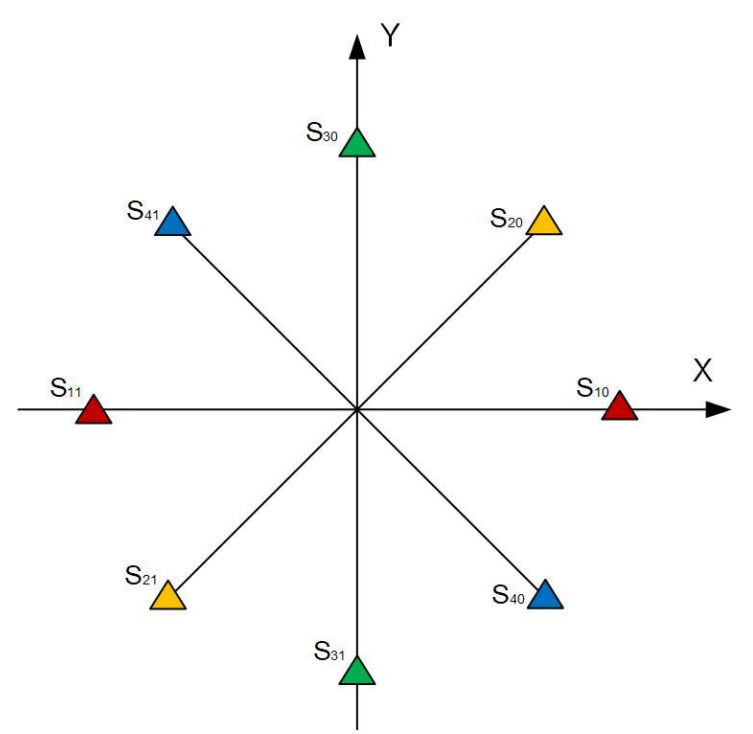

Figure 1. Sensor network configuration.

shows structural scheme of a sensor network on a plane $X Y$ consisting of four sensor pairs $B=4$.

Figure 2 shows the measurement of the UAV angular coordinates by the $b$-th sensors pair of sensor network in rectangular coordinate system $X Y Z$. UAV position is characterized by point with coordinates $(x, y, z)$. Both sensors $S_{b i}, i=\overline{0,1} \quad$ measure target bearing $\varphi_{b i}^{M}, i=\overline{0,1}$. Zero sensor $S_{b 0}$ measures also UAV elevation angle $\theta_{b 0}^{M}$. For each base, its rotation angle $\psi_{b}$ relative to the $Y$ axis is also given.

The equations of UAV angular coordinates measurement at the $k$-th step by all sensors pairs $S_{b i}, i=\overline{0,1}, b=\overline{1, B}$ of sensor network have the form

$$
\begin{aligned}
& \varphi_{b 0}^{M}(k)=\varphi_{b 0}(k)+\Delta \varphi_{b 0}(k) ; \\
& \varphi_{b 1}^{M}(k)=\varphi_{b 1}(k)+\Delta \varphi_{b 1}(k) ; \\
& \theta_{b 0}^{M}(k)=\theta_{b 0}(k)+\Delta \theta_{b 0}(k), b=\overline{1, B},
\end{aligned}
$$

where $\varphi_{b 0}(k), \varphi_{b 1}(k), \theta_{b 0}(k)$ are true UAV azimuths; $\Delta \varphi_{b 0}(k), \Delta \varphi_{b 1}(k)$, $\Delta \theta_{b 0}(k)$ - azimuths measurement errors with zero expected values and dispersions. $\sigma_{\varphi}^{2}$ and $\sigma_{\theta}^{2}$ respectively.

Taking into account the obtained angular measurements of sensor network, $B$ sets of UAV coordinates in rectangular coordinate system are determined by formulas:

$$
\begin{aligned}
& x_{b}^{M}(k)=D_{b}^{M}(k) \sin \varphi_{b 0}^{M}(k) ; \\
& y_{b}^{M}(k)=D_{b}^{M}(k) \cos \varphi_{b 0}^{M}(k) ; \\
& z_{b}^{M}(k)=D_{b}^{M}(k) \operatorname{tg} \theta_{b 0}^{M}(k), b=\overline{1, B},
\end{aligned}
$$

where $D_{b}^{M}(k)$ is projection of distance from reference receiver $S_{b 0}$ to target on $X Y$ plane, which is calculated by formula

$$
D_{b}^{M}(k)=\frac{d_{b} \sin \left(180-\left(\psi_{b}-\varphi_{b 1}^{M}(k)\right)\right)}{\sin \left(\left(\psi_{b}-\varphi_{b 1}^{M}(k)\right)-\left(\psi_{b}-\varphi_{b 0}^{M}(k)\right)\right)},
$$


$d_{b}$ is length of the $b$-th base, which is determined by formula $d_{b}=\sqrt{\left(x_{b 0}-x_{b 1}\right)^{2}+\left(y_{b 0}-y_{b 1}\right)^{2}}$.

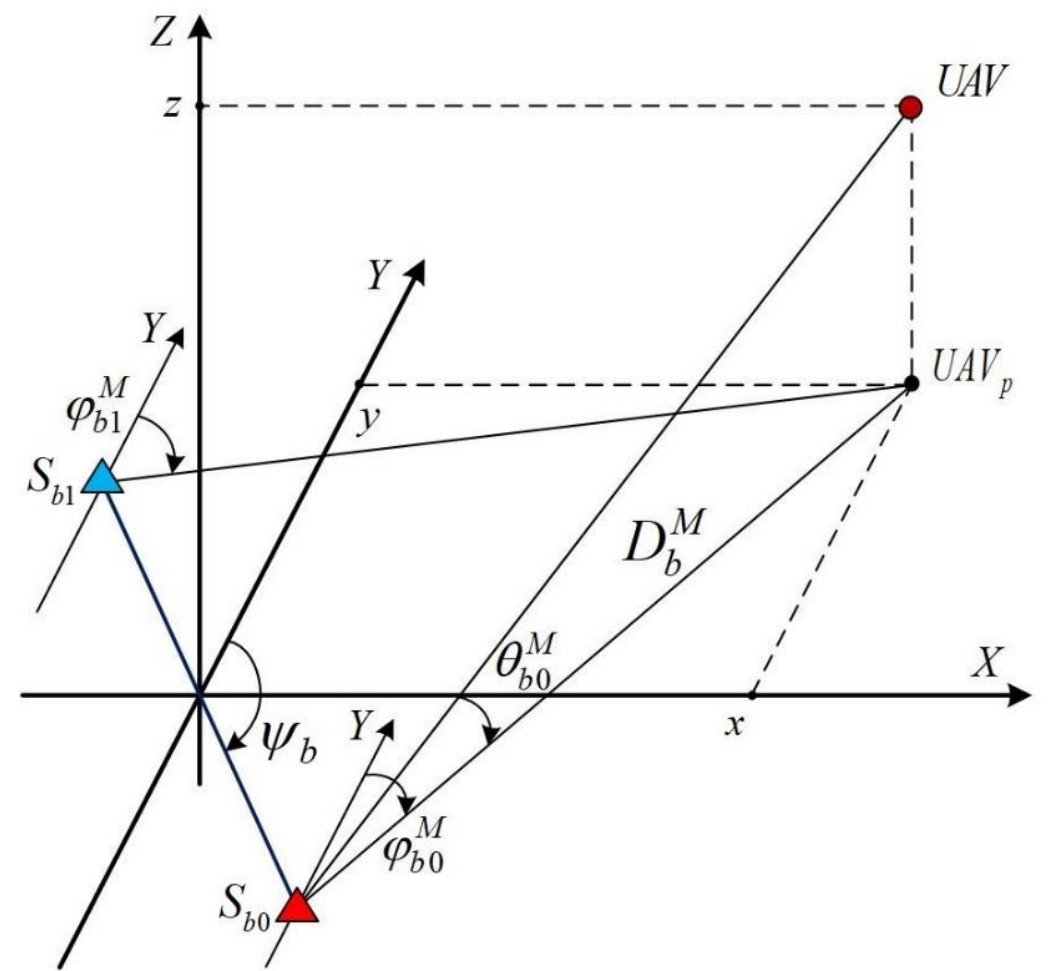

Figure 2. Measurement of the UAV angular coordinates by sensors pair $S_{b i}, i=\overline{0,1}$ of sensor network.

Equations (4)...(6) are nonlinear and describe the process of measuring UAV coordinates based on AOA-measurements of the sensor network.

UAV movement with different types of maneuver in rectangular coordinate system can be described by a discrete dynamical system with Markov switching (Tovakch \& Zhuk, 2017b):

$$
u(k)=F_{j} u(k-1)+G_{j} \omega(k), j=\overline{1, M},
$$

where $u(k)$ is state vector that includes UAV movement parameters along the axes of a rectangular coordinate system; $F_{j}, G_{j}$ are matrices that describe different movement types; $\omega(k)$ is uncorrelated sequence of Gaussian vectors with a unit correlation matrix.

To describe the UAV movement model structure type (8) corresponding to a certain maneuver type, a switching variable is used $a_{j}(k), j=\overline{1, M}$. It is a Markov chain with transition probability matrix $\Pi_{i, j}(k, k-1), i, j=\overline{1, M}$ and 
initial probabilities $p_{i}(0), i=\overline{1, M}$, which allows to take into account transitions between different types of UAV movement at random times.

Measurement equations (4)...(6) and the UAV movement model (8) are the initial ones for the synthesis of optimal and quasi-optimal trajectory filtering algorithms in a rectangular coordinate system. In the considered formulation of the problem, along with the UAV movement unknown parameters assessment, the task of recognizing its maneuver type should be solved. Therefore, the synthesized algorithms belong to the adaptive class.

\section{Synthesis of an Optimal Algorithm}

The most complete solution to the filtration problem is to determine the a posteriori probability density function (p.d.f.) of the filtered process. Based on it, estimates of unknown parameters for any loss function can be determined.

Introduce vector $\zeta_{b}(k)=\left(x_{b}^{M}(k), y_{b}^{M}(k), z_{b}^{M}(k)\right)^{T}$, which includes the UAV coordinates obtained at the $k$-th step using measurements of sensors pair $S_{b i}, i=\overline{0,1}$. UAV coordinates, obtained at the $k$-th step from all sensors pairs $S_{b i}, i=\overline{0,1}, b=\overline{1, B}$, denote as a vector $\zeta(k)=\left(\zeta_{1}(k), \ldots, \zeta_{B}(k)\right)^{T}$.

Expanded process $\left(u(k), a_{j}(k)\right)$ possesses Markov property (Zhuk, 1989). Denote the a posteriori p.d.f. of the extended process $W\left(u(k), a_{j}(k)\right)$ $=P\left(u(k), a_{j}(k) / \mathrm{Z}(k)\right)$, where $\mathrm{Z}(k)=\zeta(1), \ldots, \zeta(k)$ is obtained measurements sequence up to the $k$-th moment inclusive. Following the synthesis technique considered in (Zhuk, 1989), it can be shown that the optimal filtration algorithm can be represented in the form of two recurrence equations

$$
\begin{aligned}
& W^{*}\left(u(k), a_{j}(k)\right)=\sum_{i=1}^{M} \Pi_{i j}(k, k-1) \int_{-\infty}^{\infty} \Pi\left(u(k) / u(k-1), a_{j}(k)\right) \times \\
& \times W\left(u(k-1), a_{i}(k-1)\right) d u(k-1) ; \\
& W\left(u(k), a_{j}(k)\right)=\prod_{b=1}^{B} P\left(\zeta_{b}(k) / u(k)\right) W^{*}\left(u(k), a_{j}(k)\right) / \\
& / P(\zeta(k) / \mathrm{Z}(k-1)),
\end{aligned}
$$

where $W^{*}\left(u(k), a_{j}(k)\right)=P\left(u(k), a_{j}(k) / Z(k-1)\right) \quad$ is extrapolated p.d.f. extended process; $P\left(\zeta_{b}(k) / u(k)\right)$ is one-step likelihood function, determined based on sensor pair measurements $S_{b i}, i=\overline{0,1}$ at the $k$-th step using equations (4)...(6); $\Pi\left(u(k) / u(k-1), a_{j}(k)\right)$ is conditional p.d.f. determined using the equation (4).

Equation (9) is the optimal algorithm for extrapolating a mixed Markov process $\left(u(k), a_{j}(k)\right)$ for one step. Using relation (10), the extrapolated p.d.f. is 
corrected based on the obtained sensor network measurements $\zeta(k)$ and $a$ posteriori p.d.f. $W\left(u(k), a_{j}(k)\right)$ is determined.

Using the probability multiplication theorem, expressions (9), (10) can be represented as

$$
\begin{aligned}
& W_{j}^{*}(k)=\sum_{i=1}^{M} \Pi_{i j}(k, k-1) W_{i}(k-1) ; \\
& W_{j}^{*}(u(k))=\sum_{i=1}^{M} \Pi_{i j}(k, k-1) W_{i}(k-1) \times \\
& \times \int_{-\infty}^{\infty} \Pi\left(u(k) / u(k-1), a_{j}(k)\right) W_{i}(u(k-1)) d u(k-1) / W_{j}^{*}(k) ; \\
& W_{j}(u(k))=P(\zeta(k) / u(k)) W_{j}^{*}(u(k)) / \\
& \quad / P\left(\zeta(k) / a_{j}(k), \mathrm{Z}(k-1)\right) ; \\
& W_{j}(k)=\prod_{b=1}^{B} P\left(\zeta_{b}(k) / a_{j}(k), \mathrm{Z}(k-1)\right) W_{j}^{*}(k) / \\
& \quad / P(\zeta(k) / \mathrm{Z}(k-1)),
\end{aligned}
$$

where $W_{j}^{*}(u(k))=P\left(u(k) / a_{j}(k), \mathrm{Z}(k-1)\right), W_{j}(u(k))=P\left(u(k) / a_{j}(k), \mathrm{Z}(k)\right)$ are conditional extrapolated and a posteriori p.d.f. vector $u(k)$ provided $a_{j}(k)$; $W_{j}^{*}(k), W_{j}(k)$ are extrapolated and a posteriori probabilities $a_{j}(k)$; $P\left(\zeta(k) / a_{j}(k), \mathrm{Z}(k-1)\right)$ is conditional p.d.f. determined by the formula

$$
\begin{aligned}
& P\left(\zeta(k) / a_{j}(k), \mathrm{Z}(k-1)\right)=\int_{-\infty}^{\infty} P\left(\zeta(k) / u(k), a_{j}(k)\right) W_{j}(u(k)) d u(k) ; \\
& P(\zeta(k) / \mathrm{Z}(k-1)) \text { - determined by the formula } \\
& P(\zeta(k) / \mathrm{Z}(k-1))=\sum_{j=1}^{M} P\left(\zeta(k) / a_{j}(k), \mathrm{Z}(k-1)\right) W_{j}^{*}(k) .
\end{aligned}
$$

Initial conditions for algorithm (11) - (14) have the form $W_{i}(0)=p_{i}(0), W_{i}(u(0))=P(u(0)), i=\overline{1, M}$.

Using equations (11), (14) we calculate extrapolated $W_{j}^{*}(k)$ and $a$ posteriori $W_{j}(k)$ probabilities, and equations (12), (13) - conditional extrapolated $W_{j}^{*}(u(k))$ and a posteriori $W_{j}(u(k))$ p.d.f.. A feature of the synthesized algorithm is the inextricably linked equations of filtration and extrapolation of discrete $a_{j}(k)$ and continuous $u(k)$ components between themselves. The filtering algorithm (11)...(14) determines the structure of the optimal device. The optimal filtering device has $M$ channels, feedbacks between 
the internal channels are due to the Markov property of the discrete component $a_{j}(k)$.

Real-time implementation of the optimal algorithm (11)...(14) is difficult. Moreover, its non-linear character is primarily due to the form of the measurement equations (4)...(6).

\section{Linearization of UAV Coordinate Measurement Equations in a Cartesian Coordinate System}

We will linearize the sensor network measurement equations in a rectangular coordinate system (4)...(6). In order to reduce the notation, we omit the dependence on discrete time in expressions (4)...(6).

Expanding trigonometric functions in expression (4) into Taylor series in the vicinity of true bearing values $\varphi_{b 0}, \varphi_{b 1}$ and, limiting to linear decomposition terms, we can obtain a linearized expression describing the projection of the distance from the reference receiver $S_{b 0}$ to the target in the form (Tovkach et al. 2020)

$$
D_{b}^{M}=D_{b}+\Delta D_{b},
$$

where $D_{b}$ is the true projection value, determined by the formula (7) when substituting the true azimuths $\varphi_{b 0}, \varphi_{b 1}$ into it; $\Delta D_{b}$ is projection definition error, which is described by the expression

$$
\Delta D_{b}=c_{1} \Delta \varphi_{b 0}+c_{2} \Delta \varphi_{b 1},
$$

where $c_{1}, c_{2}$ are coefficients determined by the formulas

$$
\begin{aligned}
& c_{1}=-\frac{b_{b} \sin \left(180-\left(\alpha_{b}-\varphi_{b 1}\right)\right) \cos \left(\left(\alpha_{b}-\varphi_{b 1}\right)-\left(\alpha_{b}-\varphi_{b 0}\right)\right)}{\sin \left(\left(\alpha_{b}-\varphi_{b 1}\right)-\left(\alpha_{b}-\varphi_{b 0}\right)\right)^{2}}, \\
& c_{2}=\frac{b_{b}}{\sin ^{2}\left(\left(\alpha_{b}-\varphi_{b 1}\right)-\left(\alpha_{b}-\varphi_{b 0}\right)\right)}\left(\cos \left(180-\left(\alpha_{b}-\varphi_{b 1}\right)\right) \times\right. \\
& \times \sin \left(\left(\alpha_{b}-\varphi_{b 1}\right)-\left(\alpha_{b}-\varphi_{b 0}\right)\right)+ \\
& \left.+\sin \left(180-\left(\alpha_{b}-\varphi_{b 1}\right)\right) \cos \left(\left(\alpha_{b}-\varphi_{b 1}\right)-\left(\alpha_{b}-\varphi_{b 0}\right)\right)\right) .
\end{aligned}
$$

From formula (16) it follows that the expected values of error $\Delta D_{b}$ is zero, and its dispersion is determined by the formula

$$
\sigma_{D_{b}}^{2}=\sigma_{\varphi}^{2}\left(c_{1}^{2}+c_{2}^{2}\right) \text {. }
$$

Expanding trigonometric functions in expressions (4)...(6) in a Taylor series regarding parameters $D_{b}, \varphi_{b 0}$ and $\theta_{b 0}$ and, limiting to linear decomposition terms, it is possible to obtain linearized equations for measuring in the form 


$$
\begin{aligned}
& x_{b}^{M}=x_{b}+\Delta x_{b} ; \\
& y_{b}^{M}=y_{b}+\Delta y_{b} ; \\
& z_{b}^{M}=z_{b}+\Delta z_{b},
\end{aligned}
$$

where $x_{b}, y_{b}, z_{b}$ are true UAV coordinates; $\Delta x_{b}, \Delta y_{b}, \Delta z_{b}$ are measurement errors that are described by expressions

$$
\begin{array}{ll}
\Delta x_{b}=\alpha_{1} \Delta \varphi_{b 0}+\alpha_{2} \Delta \varphi_{b 1} ; & \\
\Delta y_{b}=\beta_{1} \Delta \varphi_{b 0}+\beta_{2} \Delta \varphi_{b 1} ; & \\
\Delta z_{b}=\gamma_{1} \Delta \varphi_{b 0}+\gamma_{2} \Delta \varphi_{b 0}+\gamma_{3} \Delta \theta_{b 0} ; & \\
\alpha_{1}, \alpha_{2}, \beta_{1}, \beta_{2}, \gamma_{1}, \gamma_{2}, \gamma_{3} \quad \text { coefficients determined by the formulas } \\
\alpha_{1}=c_{1} \sin \varphi_{b 0}+D_{b} \cos \varphi_{b 0} ; \quad \alpha_{2}=c_{2} \sin \varphi_{b 0} ; \\
\beta_{1}=c_{1} \cos \varphi_{b 0}+D_{b} \sin \varphi_{b 0} ; & \beta_{2}=c_{2} \cos \varphi_{b 0} ; \\
\gamma_{1}=c_{1} \operatorname{tg} \theta_{b 0} ; \quad \gamma_{2}=c_{2} \operatorname{tg} \theta_{b 0} ; & \gamma_{3}=D_{b} \frac{1}{\cos ^{2} \theta_{b 0}} .
\end{array}
$$

From the expressions (18)...(20) it follows that the coordinate errors have zero expected values and the correlation matrix $\mathbf{R}_{b}$, the elements of which are determined by formulas

$$
\begin{aligned}
& R_{b}(1,1)=\sigma_{b X}^{2}=\sigma_{\varphi}^{2}\left(\alpha_{1}^{2}+\alpha_{2}^{2}\right) ; \\
& R_{b}(2,2)=\sigma_{b Y}^{2}=\sigma_{\varphi}^{2}\left(\beta_{1}^{2}+\beta_{2}^{2}\right) ; \\
& R_{b}(3,3)=\sigma_{b Z}^{2}=\sigma_{\varphi}^{2}\left(\gamma_{1}^{2}+\gamma_{2}^{2}\right)+\sigma_{\theta}^{2} \gamma_{3}^{2} ; \\
& R_{b}(1,2)=R_{b}(2,1)=\sigma_{\varphi}^{2}\left(\alpha_{1} \beta_{1}+\alpha_{2} \beta_{2}\right) ; \\
& R_{b}(1,3)=R_{b}(3,1)=\sigma_{\varphi}^{2}\left(\alpha_{1} \gamma_{1}+\alpha_{2} \gamma_{2}\right) ; \\
& R_{b}(2,3)=R_{b}(3,2)=\sigma_{\varphi}^{2}\left(\beta_{1} \gamma_{1}+\beta_{2} \gamma_{2}\right) .
\end{aligned}
$$

Analysis of the accuracy characteristics of UAV coordinates determination by sensors pair $S_{1 i}, i=\overline{0,1}$ with coordinates $(100 ; 0 ; 0),(-100 ; 0$; 0 ) of sensor network is carried out using statistical modeling. The UAV was located on a circle with a radius of $1000 \mathrm{~m}$ relative to the reference origin. RMS of angular coordinate measurement errors $\sigma_{\varphi}=\sigma_{\theta}=0.4^{\circ}$.
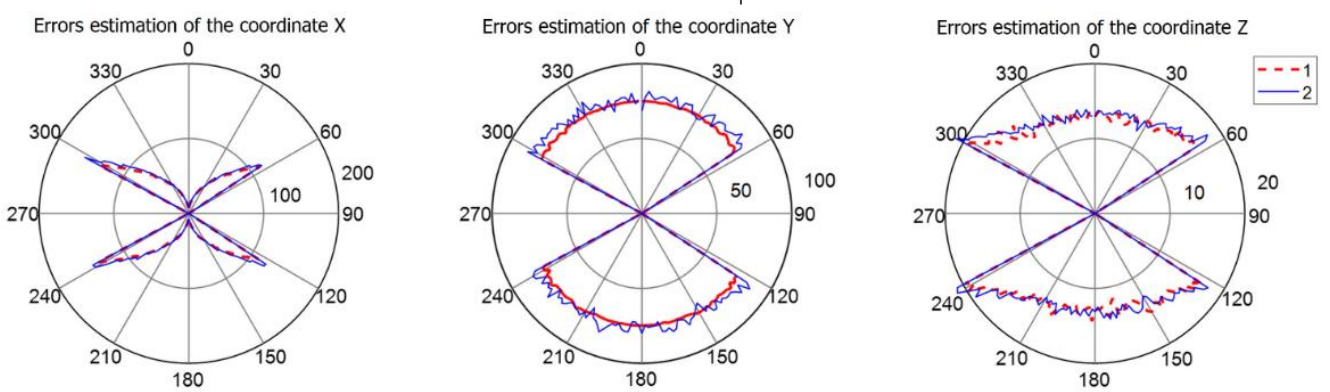

Figure 3. RMS error determining UAVs coordinate for sensor pair. 
Figure 3 shows dependences of actual RMS $\sigma_{1 X}^{M K}, \sigma_{1 Y}^{M K}$ (curves 1) error determining UAVs coordinate $(\mathrm{X}, \mathrm{Y}, \mathrm{Z})$, when using a sensor pair $S_{1 i}, i=\overline{0,1}$ obtained by Monte Carlo method. Also Figure 3 shows the dependences of theoretical RMS $\sigma_{1 X}, \sigma_{1 Y}$ (curve 2) error determining UAVs coordinate (X, Y, $\mathrm{Z}$ ), which are calculated by the formulas (21)...(23). Theoretical and actual RMS errors estimation are close, which indicates the correct calculation of accuracy characteristics.

Taking into account the UAV movement model (8), linearized equations describing the process of measuring UAV based on AOA measurements of the sensor network, has the form

$$
\zeta_{b}(k)=H u(k)+\mathrm{v}_{b}(k)+L_{b 0}, \quad b=\overline{1, B},
$$

where $v(k)=\left(\Delta x_{b}, \Delta y_{b}, \Delta z_{b}\right)$ is measurement error vector with correlation matrix $\mathrm{R}_{b}(k) ; L_{b 0}=\left(x_{b 0}, y_{b 0}, z_{b 0}\right)$ is reference sensor position; $H$ is known matrix.

\section{Synthesis of a Quasi-Optimal Algorithm}

For a linear model (8), (27), the optimal algorithm for calculating the $a$ posteriori p.d.f. $W\left(u(k), a_{j}(k)\right)$ of extended process $\left(u(k), a_{j}(k)\right)$ is also described by expressions (11)...(14). However, even in this case, the conditional a posteriori p.d.f. $W_{j}(u(k))$ are not Gaussian. To implement the optimal algorithm, it is necessary to integrate multidimensional probability densities, which leads to large computational costs and complicates its implementation in practice.

A quasi-optimal adaptive filtering algorithm can be obtained by Gaussian approximation of conditional extrapolated p.d.f. $W_{j}^{*}(u(k))$ (Zhuk, 1989). In this case, the equation for calculating the conditional extrapolated p.d.f. $W_{j}^{*}(u(k))(12)$ comes down to calculation of it's first $u_{j}^{*}(k)$ and second $P_{j}^{*}(k)$ moments by formulas (Tovkach \& Zhuk, 2019).

$$
\begin{aligned}
& u_{j}^{*}(k)=\sum_{i=1}^{M} \Pi_{i j}(k, k-1) W_{i}(k-1) F \hat{u}_{i}(k-1) / W_{j}^{*}(k) ; \\
& P_{j}^{*}(k)=\sum_{i=1}^{M} \Pi_{i j}(k, k-1) W_{i}(k-1)\left\{F_{j} \hat{P}_{i}(k-1) F_{j}{ }^{T}+G_{j} G_{j}{ }^{T}\right\} / W_{j}^{*}(k) .
\end{aligned}
$$

Equation of calculation of conditional a posteriori p.d.f. $W_{j}(u(k))(13)$ at sequentially processing of the arriving measurements $\zeta_{b}(k), b=\overline{1, B}$ comes down to calculation of it's first $\hat{u}_{j}(k)$ and second $\hat{P}_{j}(k)$ moments using the recurrent procedure (Tovkach \& Zhuk, 2017c, Tovkach \& Zhuk, 2019) 


$$
\begin{aligned}
& K_{b j}(k)=\hat{P}_{b-1 j}(k) H^{T}\left(H \hat{P}_{b-1 j}(k) H^{T}+R_{b}(k)\right)^{-1} ; \\
& \hat{u}_{b j}(k)=\hat{u}_{b-1 j}(k)+K_{j}(k)\left(\zeta_{b}(k)-H \hat{u}_{b-1 j}(k)\right) ; \\
& \hat{P}_{b j}(k)=\hat{P}_{b-1 j}(k)-K_{b j}(k) H \hat{P}_{b-1 j}(k),
\end{aligned}
$$

where $\hat{u}_{b j}(k), \hat{P}_{b j}(k)$ are expected value and correlation matrix of conditional a posteriori p.d.f. $W_{j}(u(k))$, refined by measurements $\zeta_{b}(k), b=\overline{1, B}$. Initial conditions for the procedure (30)...(32) have the form $\hat{u}_{0 j}(k)=u_{j}^{*}(k), \hat{P}_{0 j}(k)=P_{j}^{*}(k), j=\overline{1, M}, \quad$ a $\quad \hat{u}_{j}(k)=\hat{u}_{B j}(k), \hat{P}_{j}(k)=\hat{P}_{B j}(k)$, $j=\overline{1, M}$.

The filtration algorithm of discrete components doesn't change and is described by equation (11), (14). In this case conditional p.d.f. $P\left(\zeta_{b}(k) / a_{j}(k), Z(k-1)\right)=N\left(H \hat{u}_{b-1 j}(k), D_{b j}(k)\right)$ is Gaussian, and correlation matrix $D_{j}(k)$ is determined by expression

$$
D_{b j}(k)=H \hat{P}_{b-1 j}(k) H^{T}+\mathrm{R}_{b}(k) \text {. }
$$

The quasi-optimal algorithm (11), (14), (28)...(32) is nonlinear. In contrast to the optimal algorithm, only the first and second moments of conditional a posteriori distributions are calculated in its implementation. In this case, a posteriori p.d.f. $W(u(k))$ during the transition to the next filtration step is approximated by sum of $M$ Gaussian densities. Quasi-optimal filtering device (11), (14), (28)...(32) has $M$ channels and maintains the structure of the optimal device.

\section{Analysis of Efficiency of the Algorithm}

Analysis of effectiveness of developed quasi-optimal adaptive algorithm (11), (14), (28)...(32) for estimating UAV movement parameters was carried out using statistical modeling.

The sensor network (Figure 4) consists of eight sensors with coordinates: $\mathrm{S}_{10}(100 ; 0 ; 0), \mathrm{S}_{20}(70.71 ; 70.71 ; 0), \mathrm{S}_{30}(0 ; 100 ; 0), \mathrm{S}_{40}(70.71 ;-70.71 ; 0), \mathrm{S}_{11}(-$ $100 ; 0 ; 0), S_{21}(-70.71 ;-70.71 ; 14), S_{31}(0 ;-100 ; 0), S_{41}(-70.71 ; 70.71 ; 0)$.

To illustrate of algorithm operation test UAV movement trajectory was formed (Figure 4) and Table 1.

Table 1

UAV Trajectory
\begin{tabular}{|c|c|c|}
\hline Section & Interval & Type \\
\hline 1 & $1<k<42$ & uniform motion \\
\hline 2 & $43<k<45$ & maneuver \\
\hline 3 & $46<k<84$ & uniform motion \\
\hline 4 & $85<k<114$ & hanging \\
\hline 5 & $115<k<130$ & uniform motion \\
\hline
\end{tabular}


RMS of measurement errors $\sigma=0.8^{\circ}$, step of sampling $\mathrm{T}=1 \mathrm{~s}$. The simulation was carried out on a hundred realization.

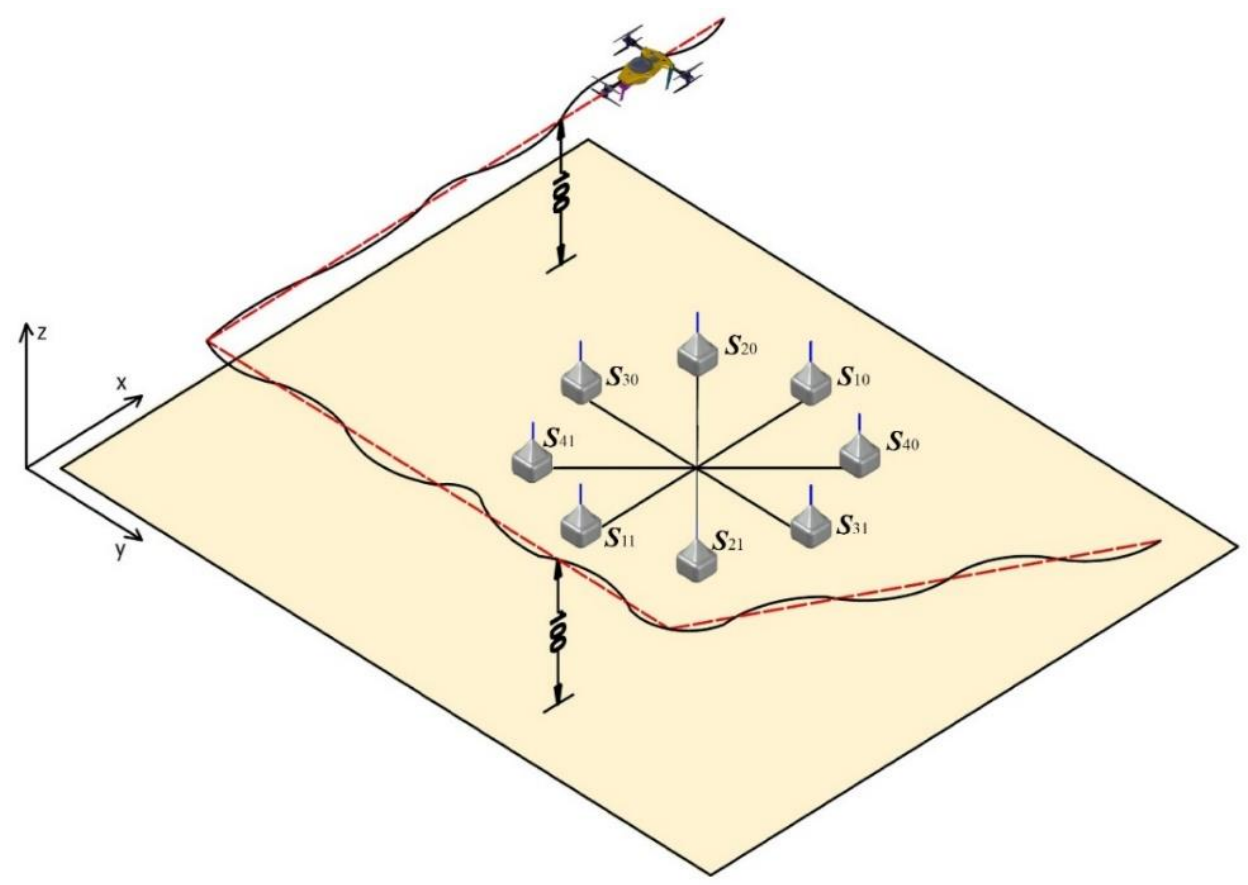

Figure 4. The configuration of the sensor network with 8 sensors and the trajectory of UAV movement.

To describe the UAV movement, we used a model with a random structure (8), which takes into account three main types of motion $M=3$ : hanging $j=1$, almost uniform motion $j=2$, maneuver $j=3$. State vector has form

$$
u^{T}(k)=(x(k), \dot{x}(k), \ddot{x}(k), y(k), \dot{y}(k), \ddot{y}(k), z(k), \dot{z}(k), \ddot{z}(k)) .
$$

where $x(k), y(k), z(k)$ are position coordinates; $\dot{x}(k), \dot{y}(k), \dot{z}(k)$ are velocities; $\ddot{x}(k), \ddot{y}(k), \ddot{z}(k)$ are accelerations.

The matrices included in the movement model (23) have the form

$$
F_{j}(k, k-1)=\left[\begin{array}{ccc}
F_{j}^{b} & 0 & 0 \\
0 & F_{j}^{b} & 0 \\
0 & 0 & F_{j}^{b}
\end{array}\right], G_{j}(k)=\left[\begin{array}{ccc}
G_{j}^{b} & 0 & 0 \\
0 & G_{j}^{b} & 0 \\
0 & 0 & G_{j}^{b}
\end{array}\right],
$$

where $F_{j}^{b}, G_{j}^{b}, j=\overline{1,3}$ have the form 


$$
\begin{gathered}
F_{1}^{b}=\left[\begin{array}{lll}
1 & 0 & 0 \\
0 & 0 & 0 \\
0 & 0 & 0
\end{array}\right], F_{2}^{b}=\left[\begin{array}{ccc}
1 & T & 0 \\
0 & 1 & 0 \\
0 & 0 & 0
\end{array}\right], F_{3}^{b}=\left[\begin{array}{ccc}
1 & T & \frac{T^{2}}{2} \\
0 & 1 & T \\
0 & 0 & 1
\end{array}\right], \\
G_{1}^{b}=\left[\begin{array}{c}
a_{1} \cdot T \\
0 \\
0
\end{array}\right], G_{2}^{b}=\left[\begin{array}{c}
\frac{a_{2} \cdot T^{2}}{2} \\
a_{2} \cdot T \\
0
\end{array}\right], G_{3}^{b}=\left[\begin{array}{c}
\frac{a_{3} \cdot T^{3}}{6} \\
\frac{a_{3} \cdot T^{2}}{2} \\
a_{3} \cdot T
\end{array}\right]
\end{gathered}
$$

$a_{1}, a_{2}, a_{3}$ - parameters characterizing the intensity of maneuver for each type of movement, which take values: $a_{1}=0.05 \mathrm{M} / \mathrm{c} ; a_{2}=0.1 \mathrm{M} / \mathrm{c}^{2} ; a_{3}=6 \mathrm{M} / \mathrm{c}^{3}$.

The initial conditions $\hat{u}_{j}(0), \hat{P}_{j}(0), j=\overline{1,3}$ for the first hypothesis $j=1$ were created on the current measurements, and for $j=\overline{2,3}$ - according to the observations at the two neighboring steps.

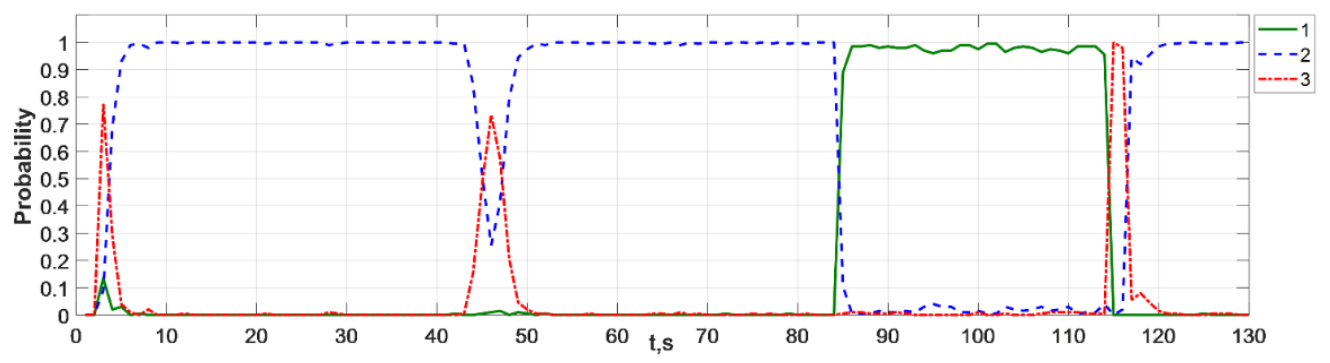

Figure 5. The probability of determining maneuver.

Figure 5 shows the dependences of movement recognition probabilities of the first (curve 1, continuous line), second (curve 2, dashed line), and third (curve 3, dash-dotted line) types obtained by the Monte Carlo method. The adaptive filter makes it possible to recognize with high probability various types of UAV movement.

Figure 6 shows theoretical RMS (curve 3) of estimation errors of the coordinates $X, Y, Z$ and also their actual mathematical expectation (curve 1) and RMS (curve 2) using the algorithm (27),(30)...(35), obtained by statistical simulation. Theoretical and actual RMS of estimation errors are close, which indicates the correct operation of algorithm. Also Figure 6 shows dependences of RMS measurement error of the UAV position which corresponds to the lower bound of Cramer-Rao (curve 4), which characterizes the potential possible accuracy of UAV coordinates determining. The use of trajectory filtering allows 
to reduce RMS of the UAV location error compared to RMS of the position error by the AOA method by 2-3 times.

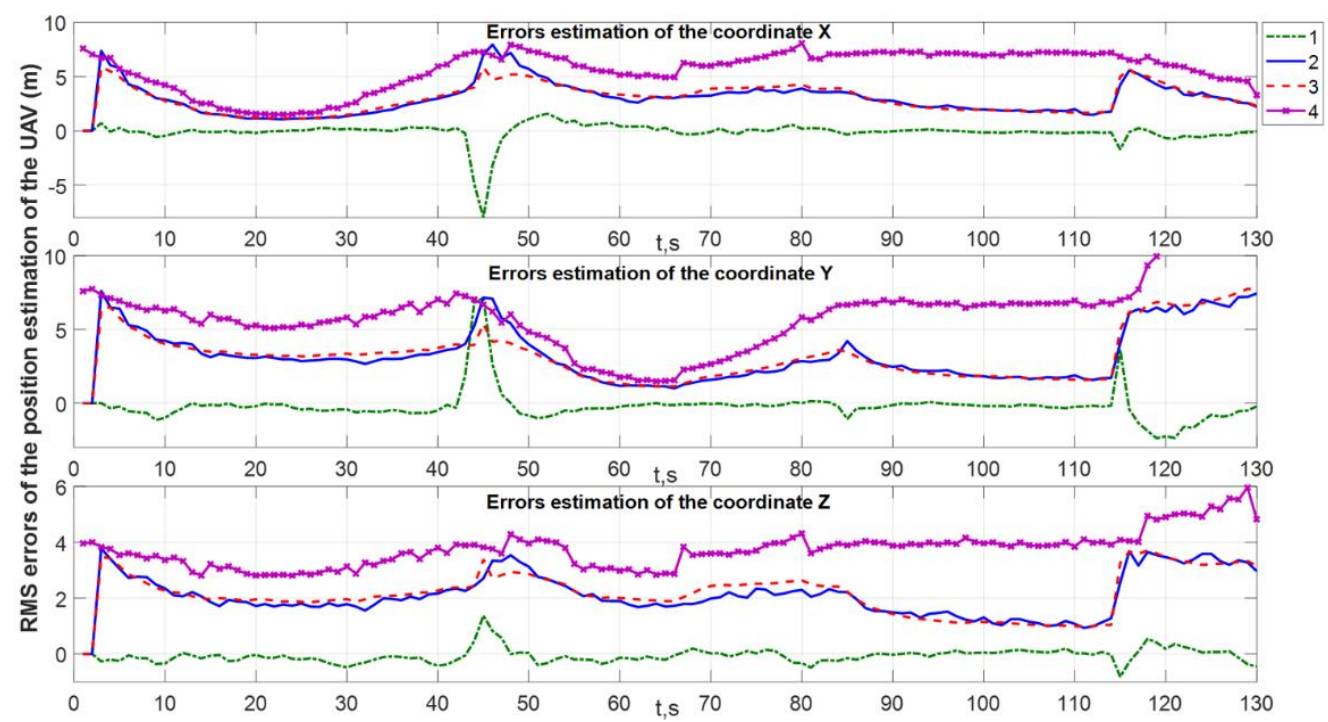

Figure 6. UAV coordinate estimation characteristics when using adaptive filter.

Also algorithms of estimating the UAV movement parameters using Kalman filters using models $j=2$ (Figure 7) and $j=3$ (Figure 8) were investigated (Tovkach \& Zhuk, 2019).

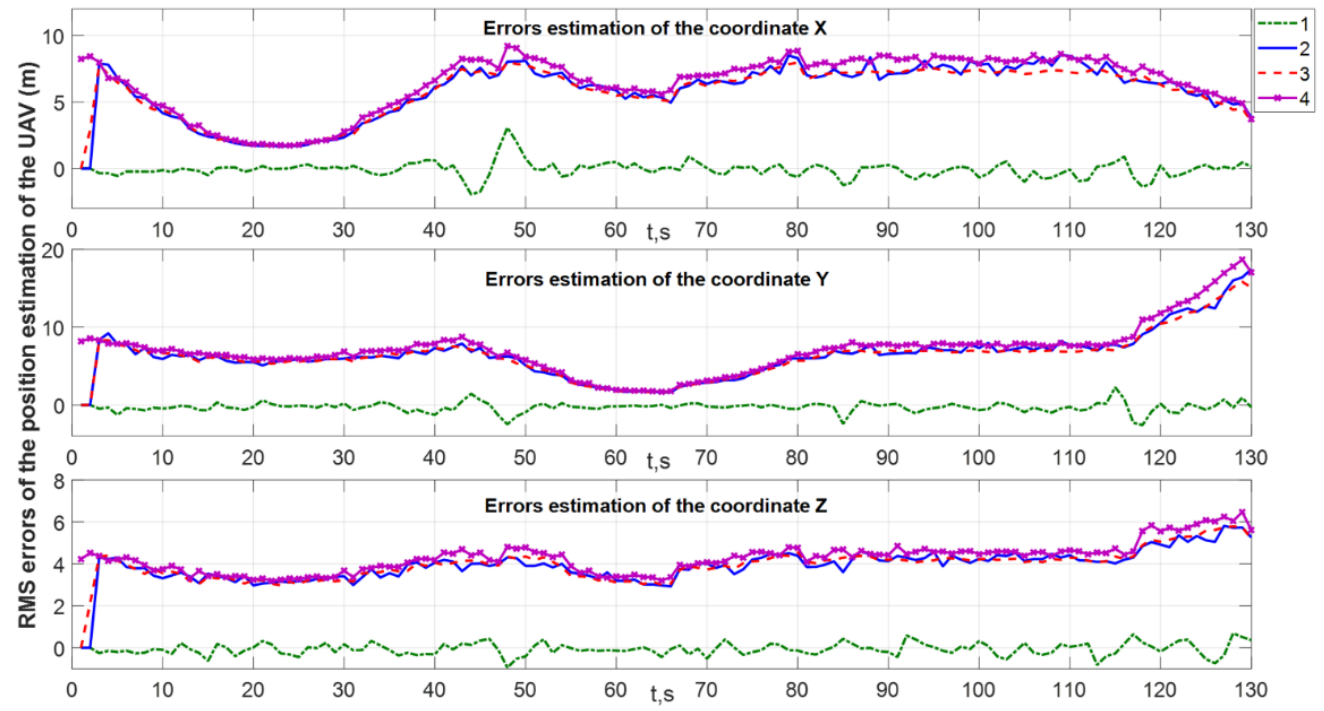

Figure 7. UAV coordinate estimation characteristics when using Kalman filter based on the model of $j=3$. 


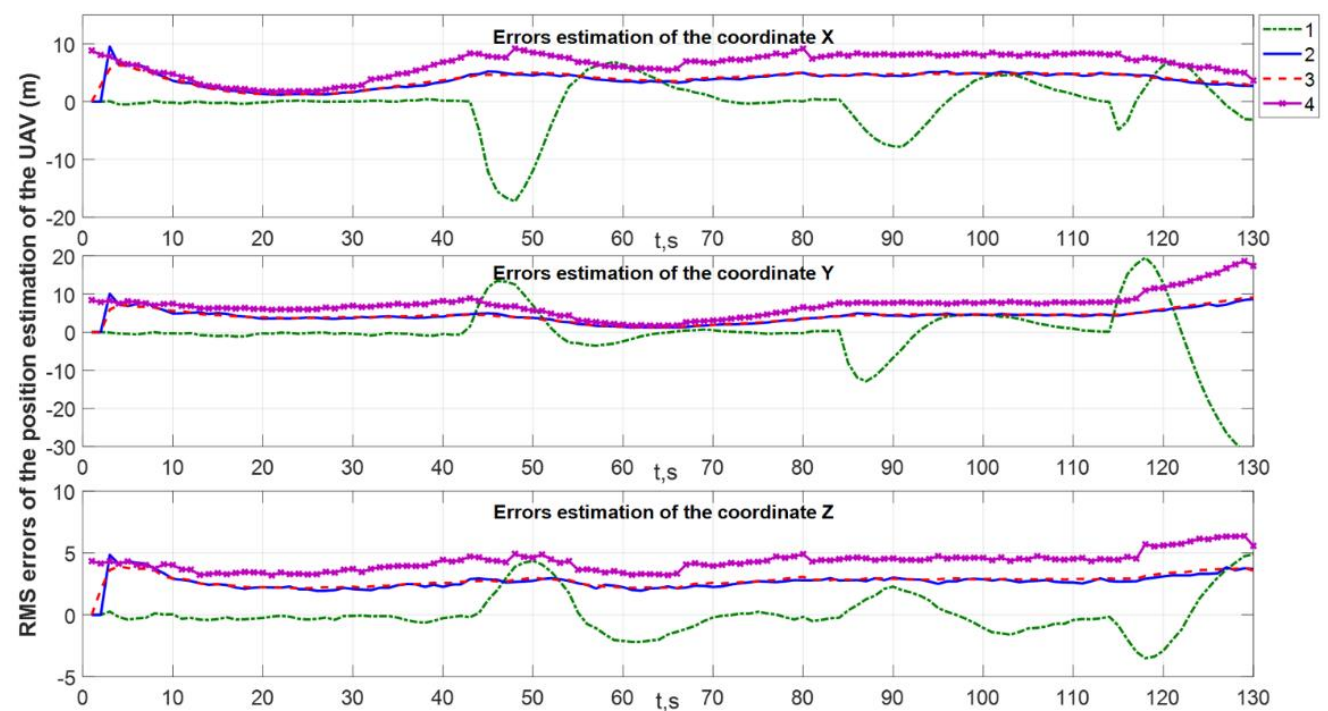

Figure 8. UAV coordinate estimation characteristics when using Kalman filter based on the model of $j=2$.

Estimates of the UAV position obtained by the Kalman filter based on the model $j=2$ contain systematic components, which is due to the presence of maneuvers. Estimates of the UAV position obtained by the Kalman filter based on the model have no systematic components, however, the RMS of the position estimation error is 2-3 times larger than theRMS of the estimation error obtained by the adaptive filter.

\section{Conclusions}

The synthesized optimal algorithm of adaptive filtering of maneuvering UAV movement parameters in a rectangular coordinate system describes the evolution of a posteriori p.d.f. of an extended mixed process and is non-linear and recurrent. A feature of the synthesized algorithm is the inextricable connection between filtration equations and extrapolation equations of discrete and continuous components. The optimal filtering device is multi-channel, feedbacks between the internal channels are due to the Markov property of the discrete component. Each channel is matched to a specific type of target movement.

In linearized measurement equations in a rectangular coordinate system, the errors in determining the UAV coordinates depend on its position in space and are correlated with each other. RMS of measurement errors in a rectangular coordinate system by a sensors pair increases as the UAV approaches the line on which they are located. The location of the sensors around the circumference allows the presence of dead zones when using the AoA method.

The synthesized quasi-optimal adaptive filtering algorithm of the maneuvering UAV movement parameters in a rectangular coordinate system is 
non-linear. In contrast to the optimal algorithm, when it is implemented, only the first and second moments of the conditional a posteriori distributions are calculated. In this case, the a posteriori p.d.f. of UAV movement parameters during the transition to the next filtering step preserves the representation in the form of a sum of Gaussian p.d.f.. The quasi-optimal filtering device (11), (14), (28)...(32) is multi-channel and preserves the structure of the optimal device.

As appears from results of modeling, application of a trajectory filtration allows to reduce RMS the UAV location error compared to RMS of the position error by the AOA method by 2-3 times. At the same time, the adaptive filter makes it possible to recognize various types of UAV movement with high probability. 


\section{References}

Amiri, R., Zamani, H., Behnia, F., \& Marvasti, F. (2016). Sparsity-aware target localization using TDOA/AOA measurements in distributed MIMO radars. ICT Express, 2(1), 23-27. https://doi.org/10.1016/j.icte.2016.02.002

Chen, C.-Y., \& Wu, W.-R. (2018). Joint AoD, AoA, and channel estimation for MIMO-OFDM Systems. IEEE Transactions on Vehicular Technology, 67(7), 5806-5820. https://doi.org/10.1109/tvt.2018.2798360

Chu, J., \& Han, W. (2019). Internet communication system protocol based on wireless sensor. Radioelectronics and Communications Systems, 62(8), 422-429. https://doi.org/10.3103/s0735272719080065

Commercial unmanned aerial vehicle market analysis - industry trends, companies and what you should know. (n.d.). Retrieved from http://www.businessinsider.com/commercial-uav-market-analysis2017-8

Hou, Y., Yang, X., \& Abbasi, Q. (2018). Efficient AoA-based wireless indoor localization for hospital outpatients using mobile devices. Sensors, 18(11), 3698. https://doi.org/10.3390/s18113698

Liu, F., Li, H., \& Yang, Z. (2018). Estimation method based on deep neural network for consecutively missing sensor data. Radioelectronics and Communications Systems, 61(6), 258-266. https://doi.org/10.3103/s0735272718060043

Peng, R., \& Sichitiu, M. L. (2006). Angle of arrival localization for wireless sensor networks. 2006 3rd Annual IEEE Communications Society on Sensor and Ad Hoc Communications and Networks. https://doi.org/10.1109/sahcn.2006.288442

Sayeed, M., \& Kumar, R. (2018). An efficient mobility model for improving transmissions in multi-UAVs enabled WSNs. Drones, 2(3), 31. https://doi.org/10.3390/drones2030031

Tang, H., Park, Y., \& Qiu, T. (2007). A TOA-AOA-based NLOS error mitigation method for location estimation. EURASIP Journal on Advances in Signal Processing, 2008(1). https://doi.org/10.1155/2008/682528

Tomic, S., Beko, M., Dinis, R., \& Bernardo, L. (2018). On target localization using combined RSS and AoA measurements. Sensors, 18(4), 1266. https://doi.org/10.3390/s18041266

Tovkach, I. O., \& Zhuk, S. Y. (2017a). Recurrent algorithm for TDOA localization in sensor networks. Journal of Aerospace Technology and Management, 9(4), 489-494. https://doi.org/10.5028/jatm.v9i4.727

Tovakch, I. O., \& Zhuk, S. Y. (2017b). Adaptive filtration of parameters of the UAV movement on data from its location calculated on the basis the time difference of arrival method. Presented at the 2017 IEEE First Ukraine Conference on Electrical and Computer Engineering (UKRCON). https://doi.org/10.1109/ukrcon.2017.8100466 
Tovkach, I. O., \& Zhuk, S. Y. (2017c). Adaptive filtration of radio source movement parameters with complex use of sensor network data based on TDOA and RSS methods. Radioelectronics and Communications Systems, 60(12), 528-537. https://doi.org/10.3103/s0735272717120020

Tovkach, I. O., Neuimin, O. S., \& Zhuk, S. Y. (2018). Filtration of parameters of the UAV movement based on the RSS-measurement at the unknown power of the transmitter. 2018 14th International Conference on Advanced Trends in Radioelecrtronics, Telecommunications and Computer Engineering (TCSET). https://doi.org/10.1109/tcset.2018.8336155

Tovkach, I. O., \& Zhuk, S. Y. (2019). Adaptive filtration of parameters of the UAV movement based on the TDOA-measurement sensor networks. Journal of Aerospace Technology and Management, 11, e3519. https://doi.org/10.5028/jatm.v11.1062

Tovkach, I., Zhuk, S., Reutska, Y., \& Neuimin, O. (2019, April). Estimation of radio source movement parameters based on TDOA- and RSSmeasurements of sensor network in presence of anomalous measurements. 2019 IEEE 39th International Conference on Electronics and Nanotechnology (ELNANO). https://doi.org/10.1109/elnano.2019.8783384

Tovkach, I. O., Zhuk, S. Y., Neuimin, O. S., \& Chmelov, V. O. (2020, April). Recurrent algorithm of passive location in sensor network by angle of arrival of a signal. 2020 IEEE 40th International Conference on Electronics and Nanotechnology (ELNANO). https://doi.org/10.1109/elnano50318.2020.9088740

Wallace, R. J., \& Loffi, J. M. (2015). Examining unmanned aerial system threats \& defenses: A conceptual analysis. International Journal of Aviation, Aeronautics, and Aerospace, 2(4). http://dx.doi.org/10.15394/ijaaa.2015.1084.

Xu, J., Ma, M., \& Law, C. L. (2008). AOA cooperative position localization. Presented at the IEEE GLOBECOM 2008 - 2008 IEEE Global Telecommunications Conference. https://doi.org/10.1109/glocom.2008.ecp.720

Yao, B., Wang, W., Han, W., \& Yin, Q. (2014). Distributed angle estimation by multiple frequencies synthetic array in wireless sensor localization system. IEEE Transactions on Wireless Communications, 13(2), 876887. https://doi.org/10.1109/twc.2013.120713.130526

Zhang, W., Yin, Q., Chen, H., Gao, F., \& Ansari, N. (2013). Distributed angle estimation for localization in wireless sensor networks. IEEE Transactions on Wireless Communications, 12(2), 527-537. https://doi.org/10.1109/twc.2012.121412.111346

Zhang, X., Huang, J., Wang, Y., \& Zhou, Y. (2018). An efficient estimator for target localization in a multistation redundancy system without matrix 
inversion. Journal of Sensors, 2018, 1-12.

https://doi.org/10.1155/2018/6362939

Zhang, W., Yin, Q., Chen, H., Gao, F., \& Ansari, N. (2013). Distributed angle estimation for localization in wireless sensor networks. IEEE Transactions on Wireless Communications, 12(2), 527-537. https://doi.org/10.1109/twc.2012.121412.111346

Zhang, R., Liu, J., Du, X., Li, B., \& Guizani, M. (2018). AOA-based threedimensional multi-target localization in industrial WSNs for LOS conditions. Sensors, 18(8), 2727. https://doi.org/10.3390/s18082727

Zhuk, S. Y. (1989). Synthesis of digital detector-meters for mixed Markovian processes. Radioelectronics and Communications Systems, 32(11), 2934. https://doi.org/10.3103/S073527271989110063

Zhuk, S. Y., Tovkach, I. O., \& Reutska, Y. Y. (2019). Adaptive filtration of radio source movement parameters based on sensor network TDOA measurements in presence of anomalous measurements.

Radioelectronics and Communications Systems, 62(2), 61-71. https://doi.org/10.3103/s073527271902002x

Zhuk, S. Y., Tovkach, I. O., \& Reutska, Y. Y. (2018, September). Adaptive filtration of radio source movement parameters based on the RSSmeasurement sensor networks in the presence of anomalous measurements. 2018 International Conference on Information and Telecommunication Technologies and Radio Electronics (UkrMiCo). https://doi.org/10.1109/ukrmico43733.2018.9047600 